\title{
Bluino: Blind Arduino Sensor to Assist Students with Visual Impairment
}

\author{
Rossy Andini Herindra Putri ${ }^{1}$, Annisa Fadhila Nur Fikriah ${ }^{2}$, Diana Ayu Latifah ${ }^{3}$, Shafa \\ Rihadatul Aisy ${ }^{4}$, Eliyawati ${ }^{5}$, Eka Cahya Prima ${ }^{6}$ \\ \{ekacahyaprima@upi.edu ${ }^{6}$ \} \\ Department of Science Education, Faculty of Mathematics and Science Education, Universitas \\ Pendidikan Indonesia, Bandung, Indonesia ${ }^{1,2,3,4,5,6}$
}

\begin{abstract}
People with visual impairment are part of our society who have the same rights as a citizen which has limited mobility. They need technology to help navigation. Arduino is a microcontroller-based technology that can be used as walking assistance. We hereby propose 'Bluino' as walking support for visual impairment students. It is equipped with an ultrasonic sensor HC-SR04 to detect an object within $50 \mathrm{~cm}$, which will trigger the buzzer to produce sound. Bluino is packed into a custom box with a strap placed on the user's leg. Three students have evaluated this device. The result shows that the tool can detect an object or obstacle in front of them so they can avoid it. The sensor can detect an object up to 4 meters and 15 degrees measuring angle. They suggested improving Bluino by simplifying the model and enabling the sensor to detect holes.
\end{abstract}

Keywords: Visual Impairment, Bluino, Arduino, Ultrasonic Sensor

\section{Introduction}

In life, we will never get perfection, including about the human birth with the thing that could be said as less perfect due to something that might not exist or not functioning correctly which also called 'disability.' People with disabilities are also part of our society who have the same rights as a citizen and the same degree as human beings created by God. One example of disabilities are people with visual impairments. They are a part of society that has limited mobility in an environment and social life. Apart from the acceptance from the social context, it is also essential for visually impaired people to receive facilities that can support them in doing daily activities. Now people can take a lot of advantage from the development of technology [1]. People with visual impairments, when they will go somewhere, they have to be accompanied by someone as a navigator or using a stick which facilitates them to their destination. However, the stick that was carried was only to find out what was in front of the stick. Therefore, instead of using the stick as a navigator, the improvement of technology has the potential to construct an assistance product for visual impairment people by using a microcontroller such as Arduino [2-6].

Arduino is an open-source electronics platform based on easy-to-use hardware and software able to read inputs - light on a sensor and turn it into an output - activating a motor [2]. Arduino can be used as a system that helps people or makes a robotic such as Self Tying Shoes and Home Security system. Arduino is also used as a system for making a product of technology to help people with visual impairments, the example of the products that used an Arduino is Blind Stick 
Navigator, Sensor-based Assistive Device, Object Detection System with Arduino, and Smart Walking Stick Using Ultrasonic Sensor and Arduino [2-6].

With that in mind, we made Bluino which stands for Blind Sensor Arduino Based. Bluino aims to help students with visual impairment to walk without using a stick. Bluino uses an ultrasonic sensor to detect an object within a certain distance. Then it sends the audio signal to warn the user when an object is detected. So, the user is aware of it and able to avoid the object. Besides helping students with visual impairment, Bluino is easy to make. Moreover, it can be used in the education class. However, several things need to be improved, such as the size, the direction of detection, and the angle of measurement.

\section{Method}

\subsection{Materials}

In this research, we need several materials which are Arduino Uno microcontroller, an ultrasonic sensor HC-SR04 module, buzzer, $9 \mathrm{~V}$ battery, $9 \mathrm{~V}$ battery snap connector, male to the male cable connector, USB cable, strap and a custom-made box as shown in Figure 1. Arduino Uno board uses integrated circuit microcontroller Atmega328. It has 14 digital input/output and six analog inputs [2]. Ultrasonic HC-SR04 is a non-contact sensor that uses the ultrasonic wave to measure distance. It has a wave transmitter and receiver [3]. The buzzer is an audio signal device. It usually used as an alarm or timer device as seen in Fig. 1.

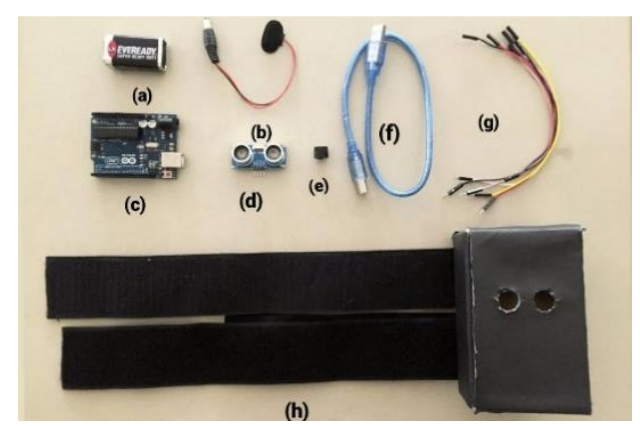

Fig. 1. Materials used to construct the Bluino prototype. The materials are (a) 9 -volt battery, (b) $9 \mathrm{~V}$ battery snap connector, (c) Arduino Uno microcontroller, (d) ultrasonic sensor HC-SR04 module, buzzer, (e) buzzer, (f) USB cable, (g) male to male cable connector and (h) custom-made box with strap.

\subsection{Coding}

The first step of making the Bluino is to make the script or code to be uploaded to the board so that we can control the device. First, we initialized ultrasonic HC-SR04 pins in the code. After that, we initialized the buzzer pin. The last, we initialized variable which calculates the distance of the objects. The equation for distance is $s=v$.t. Since the time that recorded is the time to transmit and to travel back, then the equation is $s=v \cdot \frac{t}{2}$. Below is the code for Bluino. 


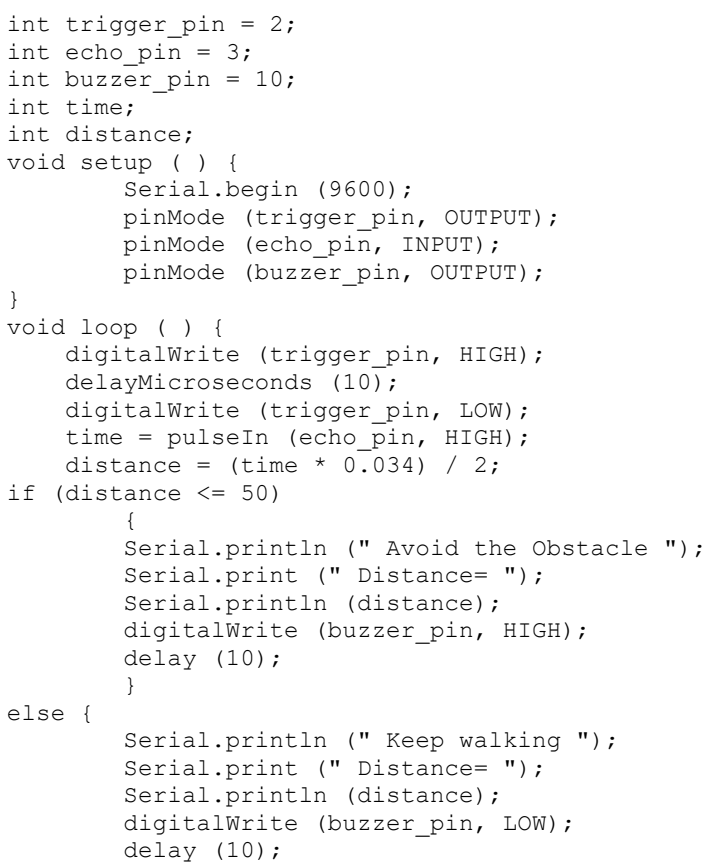

The first part of the code is where we initialized the ultrasonic sensor and buzzer. In the void set up, we set trigger pin in the ultrasonic sensor as output as it transmits ultrasonic wave, the echo pin in the ultrasonic sensor as input because it receives the ultrasonic wave which travels back after hitting an object. We also set the buzzer pin as output because it produces sound. In the void loop, we applied a distance equation to get the distance. The last part of the code is where we make a condition. If the range of the object is less than $50 \mathrm{~cm}$, the buzzer will be triggered on so that means the user needs to avoid the object. If the length of the object is more than $50 \mathrm{~cm}$, the bell will not be triggered, so that means it is safe to keep walking.

\subsection{Experiment Stage}

Bluino stands for Blind Sensor Arduino-based. It is a device which aims to help students with visual impairment to walk, by detecting object or obstacle in front of them so they can avoid it. The idea is that the device will send an audio signal if there is an object within a certain distance in front of the user. To do that, we construct the Bluino following the scheme shown in Figure 2. Then we put the device into the custom box. The device is arranged so that the sensor is facing forward and the buzzer is facing upward. We have four main components in this device. The first is Arduino as the microcontroller. The second is the ultrasonic sensor as the input. The sensor can detect an object up to 4 meters, and it has 15 degrees measuring angle [1]. With this specification, the sensor has a blind spot, as shown in Figure 3. The third principal component is buzzer as the audio signal output, and the last is the battery as the high mobility power supply. When the device is in used, the ultrasonic sensor will transmit ultrasonic wave, which will travel back when it hit an object. By this, the distance between the device and the object is recorded. When the range is less than $50 \mathrm{~cm}$, the buzzer will be triggered on so the user 
knows that there is an object less than $50 \mathrm{~cm}$ at the front and so the user needs to change direction to avoid tripping or hit the object and continue to walk straight when there is no sound made by the buzzer as shown in Figure 2 and Figure 3.

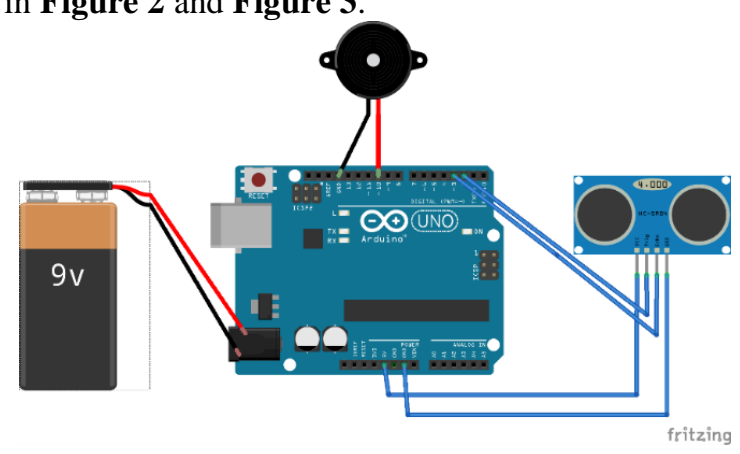

Fig. 2. Schematic view of Bluino.

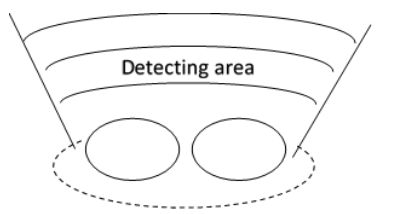

Blind spot

Fig. 3. Blind Spot of the ultrasonic sensor.

\section{Result and Discussion}

The ultrasonic sensor emits an ultrasonic wave from the trigger which comes back after hitting the object, and the echo receives it. The echo will then tell us the distance traveled in microseconds. After that, the trigger makes the buzzer beeps and warns the person about the range that set before.

Figure 4 shows the Bluino unit constructed by following the scheme in Figure 2. Meanwhile, Figure 5 and Figure 6 show the device after it is packed into the custom box and how the device looks, respectively. The device is placed on the foot just above the ankle or knee, and the strap helps us to keep the device in place. When the device is on, the buzzer turns on automatically when there is an object within $50 \mathrm{~cm}$. It also stops automatically when the user turns around to avoid the object. Figure 7 shows the procedure for constructing BLUINO.

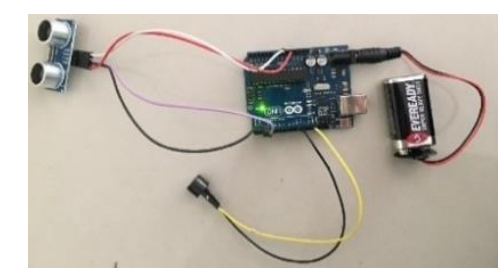


Fig. 4. Bluino unit.

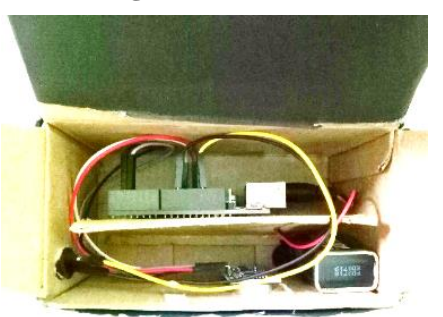

Fig. 5. Bluino unit inside the custom box.

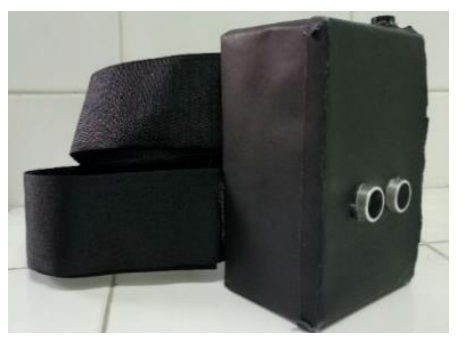

Fig. 6. Final Product of the device.

When we first try it. The device works well but still, sometimes the buzzer is on and making sound signal even though there is no object within $50 \mathrm{~cm}$ in front of the device. After evaluating it, we found the problem is the male-female cable connector because it is not stable. So, we changed the cable and the device works. We also record the serial monitor of Bluino shown in Figure 8. The graph shows that the distance between the user and the object is decreasing. This means that the user keeps walking. But every time the length of the object is less than $50 \mathrm{~cm}$, the graph change and it shows that the gap between the object is increasing. That is because the buzzer beeps and warns the user that there is an object within $50 \mathrm{~cm}$. Even so, the ultrasonic sensor still has some errors. The delay of the ultrasonic sensor that is set in Bluino is 10 . This means that this sensor has a $5 \mathrm{~ms}$ time response error. This sensor that sends out an 8 cycle burst of ultrasound at $40 \mathrm{kHz}$ also has $\pm 0.5 \mathrm{~Hz}$ error.

This product then tried and evaluated by three students with a visual impairment from Wiyata Guna Foundation. They put the device on and walks around under our supervision in turns. The students with visual impairment have a similar opinion about this product. Words like enhance, expand, and improvement of the product are three common things that included in what they thought.

According to our first statement that this product is likely a core idea to other something new. Because the idea here is to emphasize it, we can do multiple modifications to Bluino. The primary thing that can be improved according to the evaluator is to make it smaller by using Arduino Nano rather than Arduino Uno. By this, we can even make it look more attractive, like turning it into a bracelet or watch or only just something that not too eye-catching for them to use. The delay of the sensor should be decreased, so the detection can trigger the buzzer to beep and warn them faster.

Another suggestion that could be considered an enhancement for the product is the direction and range of the device since it can only detect the front side with no more than $15^{\circ}$ angle. For further research, the device is expected to identify all directions, and the measured angle could be increased to minimalize the blind spot. More important is that the tool can detect a hole to 
prevent the user from falling. This can be tackled by using more than one sensor with the audible audio signal. So that the user can be aware of the direction of the object.

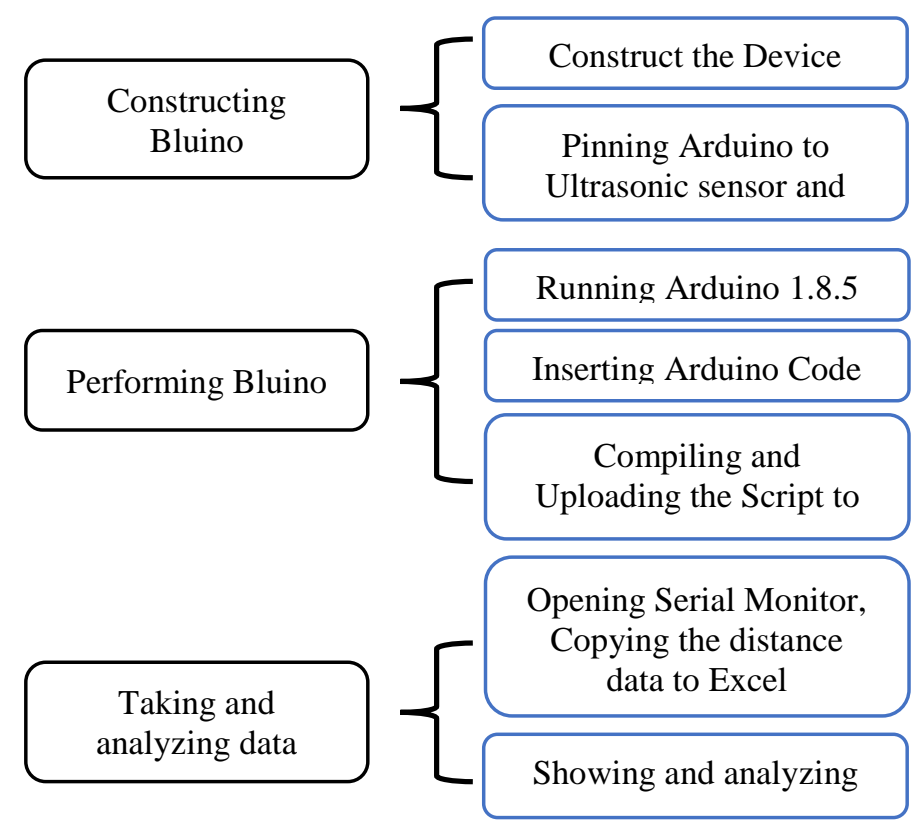

Fig. 7. The complete procedure of Bluino usage starting from constructing the device, performing the device, to taking and analyzing data.

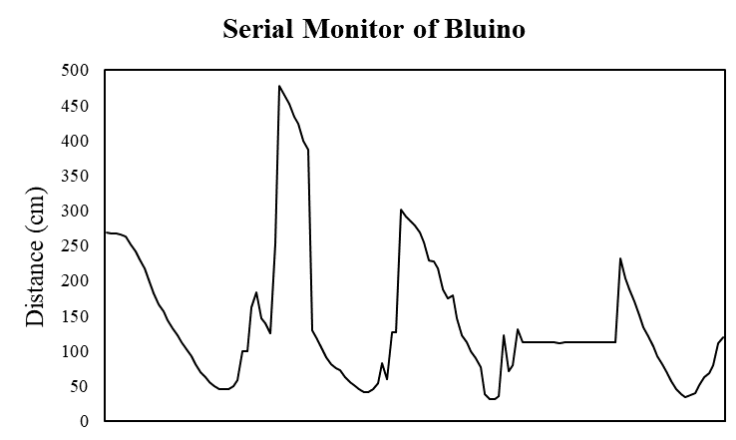

Fig. 8. Serial monitor of Bluino when it is used to assist walking. 


\section{Conclusion}

This paper has shown that Bluino can be used to help visually impaired people by using the ultrasonic sensor to detect object distance and send an audio signal to warn the user. Bluino itself has a simple schematic, so it was not very difficult for us to make it. Another advantage of this device is that it is easy to use. However, the ultrasonic sensor that we used in Bluino can only detect objects in front of the sensor and within the $15^{\circ}$ angle range.

For future references, we highly encouraged to decrease the device size by using Arduino Nano instead of Arduino Uno. Using more than one sensor is better instead of only relying on one sensor. It is preferably using a sensor that can detect holes with a minimum blind point. Moreover, it is recommended to modify the device into accessories to ensure that the user feels comfortable and making it less eye-catching. Most importantly, the delay of this device should be decreased to warn the user faster and prevent them from tripping or falling.

Acknowledgments. We would also like to thank the laboratory assistant of IPSE's Dry Laboratory; Mr. Latief Budiana, for his help in offering us the equipment needed for this project and allowing us to use the laboratory.

\section{References}

[1] Zhou, L., Parker, A. T., Smith, D. W., \& Griffin-Shirley, N.: Assistive technology for students with visual impairments: Challenges and needs in teachers' preparation programs and practice. Journal of Visual Impairment \& Blindness, 105(4), pp. 197-210 (2011)

[2] Warren, J. D., Adams, J., \& Molle, H.: Arduino for robotics. Arduino robotics, pp. 51-82. Apress, Berkeley, CA (2011)

[3] Araújo, A., Portugal, D., Couceiro, M. S., \& Rocha, R. P.: Integrating Arduino-based educational mobile robots in ROS. Journal of Intelligent \& Robotic Systems, 77(2), pp. 281-298 (2015).

[4] Prima, E. C., Munifaha, S. S., Salam, R., Aziz, M. H., \& Suryani, A. T.: Automatic water tank filling system controlled using ArduinoTM based sensor for home application. Procedia Engineering, 170, pp. 373-377 (2017)

[5] Prima, E. C., Karim, S., Utari, S., Ramdani, R., Putri, E. R. R., \& Darmawati, S. M.: Heat Transfer Lab Kit using Temperature Sensor based ArduinoTM for Educational Purpose. Procedia Engineering, 170, pp. 536-540 (2017)

[6] Mohapatra, B. N., Mohapatra, R., \& Panda, P.: Path Guidance System For Blind People. International Journal of Open Information Technologies, 7(5), pp. 29-32 (2019) 